doi:10.13108/2021-13-1-85

\title{
STUDY OF APPROXIMATE SOLUTION TO INTEGRAL EQUATION ASSOCIATED WITH MIXED BOUNDARY VALUE PROBLEM FOR LAPLACE EQUATION
}

\author{
E.H. KHALILOV, M.N. BAKHSHALIYEVA
}

\begin{abstract}
We consider an approximate solution of the integral equation arising after reduction of a mixed problem for the Laplace equation. The main advantage of applying the method of integral equations to studying external boundary value problems is that such approach allows one to reduce the problem posed in an unbounded domain to a problem in a domain of a smaller dimension. In the work we study an approximate solution to the integral equation, to which the mixed problem for the Laplace equation is reduced. We seek its solution as a combination of logarithmic single layer potentials and double layer potential, we reduce the problem to an integral equations depending not only on the operators generated by the logarithmic potentials but also on the composition of such operators. We prove that the obtained integral equation has the unique solution in the space of continuous functions. Since the integral equations can be solved in the closed form only in very rare cases, it is of a high importance to develop approximate methods for solving integral equations and give their appropriate theoretical justification. We partition a curve into elementary parts and by certain nodes we construct quadrature formulae for a class of curvilinear potentials and for the composition of the integrals generated by logarithmic potentials and we also estimate the errors of these formulae. Employing these quadrature formulae, the obtained integral equation is replaced by the system of algebraic equations. Then by means of Vainikko's convergence theorem for linear operator equations, we establish the existence and uniqueness of solutions to this system. We prove the convergence of the obtained system of algebraic equations to the values of the exact solution of the integral equation at the chosen nodes. Moreover, we find the convergence rate of this method. As a result, we find a sequence converging to the solution of the mixed boundary value problem for the Laplace equation and its convergence rate is known.
\end{abstract}

Keywords: curvilinear integral, integral equation method, collocation method, mixed boundary value problem, Laplace equation.

Mathematics Subject Classification: 45E05, 31B10

\section{INTRODUCTION AND FORMULATION OF PROBLEM}

Let $D \subset \mathbb{R}^{2}$ be a bounded domain with a twice differentiable boundary $L$.

We consider a mixed boundary value problem for the Laplace equation: find a function $u \in C^{(2)}\left(\mathbb{R}^{2} \backslash \bar{D}\right) \bigcap C\left(\mathbb{R}^{2} \backslash D\right)$ possessing a normal derivative in the sense of the uniform convergence, satisfying the Laplace equation $\Delta u=0$ in $\mathbb{R}^{2} \backslash \bar{D}$, the Sommerfeld radiation

E.H. Khalilov, M.N. Bakhshaliyeva, Study of approximate solution to integral equation ASSOCIATED WITH MIXED BOUNDARY VALUE PROBLEM FOR LAPLACE EQUATION.

(C) Khalilov E.H., Bakhshaliyeva M.N. 2021.

Submitted May 6, 2020. 
condition

$$
\left(\frac{x}{|x|}, \operatorname{grad} u(x)\right)=o\left(\frac{1}{|x|^{\frac{1}{2}}}\right), \quad x \rightarrow \infty,
$$

uniformly over all directions $x /|x|$, and the boundary condition

$$
\frac{\partial u(x)}{\partial \vec{n}(x)}+\lambda u(x)=f(x) \quad \text { on } \quad L,
$$

where $\vec{n}(x)$ is the unit normal vector at a point $x \in L$, the symbol $\lambda$ denotes a given number, while $f$ stands for a given continuous function on $L$. One of the methods of solving a mixed boundary value problem for the Laplace equation is reducing it to an integral equation. It is known that the main advantage of applying the method of integral equations to studying the outer boundary value problems is that such approach allows one to reduce the problem posed for an unbounded domain to a problem in a bounded domain of a smaller dimension.

Let $\Phi(x, y)$ be a fundamental solution of the Laplace equation, $v(x, \rho)$ be the logarithmic single layer potential, while $w(x, \rho)$ be the double layer logarithmic potential, that is,

$$
\begin{aligned}
& \Phi(x, y)=\frac{1}{2 \pi} \ln \frac{1}{|x-y|}, \quad x, y \in \mathbb{R}^{2}, \quad x \neq y, \\
& v(x, \rho)=\int_{L} \Phi(x, y) \rho(y) d L_{y}, \quad w(x, \rho)=\int_{L} \frac{\partial \Phi(x, y)}{\partial \vec{n}(y)} \rho(y) d L_{y} .
\end{aligned}
$$

It is obvious that then

$$
w(x, v)=\int_{L} \frac{\partial \Phi(x, y)}{\partial \vec{n}(y)}\left(\int_{L} \Phi(y, t) \rho(t) d L_{t}\right) d L_{y}, \quad x \in \mathbb{R}^{2} .
$$

Taking into consideration the limiting values of the logarithmic potentials and proceeding as in work [1], it is easy to show that the function

$$
u(x)=v(x, \rho)+i \mu w(x, v), \quad x \in \mathbb{R}^{2} \backslash \bar{D},
$$

where $\mu \neq 0$ is an arbitrary real number solves the mixed boundary value problem for the Laplace equation if the density $\rho$ is a solution to a uniquely solvable integral equation

$$
\rho+A \rho=\varphi,
$$

where

$$
\begin{aligned}
& A=-(2+i \mu)^{-1}(2 \tilde{K}+4 i \mu R+\lambda(2+i \mu) S+4 i \lambda \mu Q), \\
& \varphi=-4(2+i \mu)^{-1} f, \\
& (S \rho)(x)=2 \int_{L} \Phi(x, y) \rho(y) d L_{y}, \quad x \in L, \\
& (\tilde{K} \rho)(x)=2 \int_{L} \frac{\partial \Phi(x, y)}{\partial \vec{n}(x)} \rho(y) d L_{y}, \quad x \in L, \\
& (Q \rho)(x)=\int_{L} \frac{\partial \Phi(x, y)}{\partial \vec{n}(y)}\left(\int_{L} \Phi(y, t) \rho(t) d L_{t}\right) d L_{y}, \quad x \in L, \\
& (R \rho)(x)=\int_{L} \frac{\partial \Phi(x, y)}{\partial \vec{n}(x)}\left(\int_{L} \frac{\partial \Phi(y, t)}{\partial \vec{n}(y)} \rho(t) d L_{t}\right) d L_{y}, \quad x \in L .
\end{aligned}
$$


Since integral equations are solved in closed form in very rare cases, it is highly important to develop approximate methods of solving integral equations with a corresponding theoretical justification. We note that a series of works [2]-[5] was devoted to studying approximate solutions to integral equations associated with various boundary value problems. However, the approximate solving of integral equation (1.1), to which the mixed boundary value problems for the Laplace equation is reduced, has not been studied yet. Our work is devoted to covering this gap.

\section{Construction of QuAdrature FORMula}

Assume that the curve $L$ is given by the parametric equation $x(t)=\left(x_{1}(t), x_{2}(t)\right), t \in[a, b]$. We partition the segment $[a, b]$ into

identical parts:

$$
n>2 M_{1} \frac{b-a}{d}
$$

$$
t_{k}=a+\frac{(b-a) k}{n}, \quad k=\overline{0, n}
$$

where [6, Ch. VI]

$$
M_{1}=\max _{t \in[a, b]} \sqrt{\left(x_{1}^{\prime}(t)\right)^{2}+\left(x_{2}^{\prime}(t)\right)^{2}}<+\infty
$$

and $d$ is a standard radius [6, Ch. I], [7, Ch. V]. As nodes we choose $x\left(\tau_{k}\right), k=\overline{1, n}$, where

$$
\tau_{k}=a+\frac{(b-a)(2 k-1)}{2 n} \text {. }
$$

Then the curve $L$ is partitioned into elementary parts: $L=\bigcup_{l=1}^{n} L_{l}$, where

$$
L_{k}=\left\{x(t): \quad t_{k-1} \leqslant t \leqslant t_{k}\right\} .
$$

It is known that 8

(1) for each $k \in\{1,2, \ldots, n\}$, the relation holds: $r_{k}(n) \sim R_{k}(n) 1$, where

$$
\begin{aligned}
& r_{k}(n)=\min \left\{\left|x\left(\tau_{k}\right)-x\left(t_{k-1}\right)\right|,\left|x\left(t_{k}\right)-x\left(\tau_{k}\right)\right|\right\}, \\
& R_{k}(n)=\max \left\{\left|x\left(\tau_{k}\right)-x\left(t_{k-1}\right)\right|,\left|x\left(t_{k}\right)-x\left(\tau_{k}\right)\right|\right\} ;
\end{aligned}
$$

(2) for each $k \in\{1,2, \ldots, n\}$ the inequality $R_{k}(n) \leqslant \frac{d}{2}$ holds;

(3) for all $k, j \in\{1,2, \ldots, n\}$ the relation $r_{j}(n) \sim r_{k}(n)$ holds;

(4) $r(n) \sim R(n) \sim \frac{1}{n}$, where

$$
R(n)=\max _{k=1, n} R_{k}(n), \quad r(n)=\min _{k=1, n} r_{k}(n) .
$$

Lemma $2.1([3],[9])$. There exist constants $C_{0}^{\prime}>0$ and $C_{1}^{\prime}>0$ independent of $n$, such that for all $k, j \in\{1,2, \ldots, n\}, j \neq k$ and $y \in L_{j}$ the following inequality holds:

$$
C_{0}^{\prime}\left|y-x\left(\tau_{k}\right)\right| \leqslant\left|x\left(\tau_{j}\right)-x\left(\tau_{k}\right)\right| \leqslant C_{1}^{\prime}\left|y-x\left(\tau_{k}\right)\right| .
$$

For a function $\varphi(x) \in C(L)$ we introduce a continuity modulus of form

$$
\omega(\varphi, \delta)=\delta \sup _{\tau \geqslant \delta} \frac{\bar{\omega}(\varphi, \tau)}{\tau}, \quad \delta>0
$$

where

$$
\bar{\omega}(\varphi, \tau)=\max _{\substack{|x-y| \leqslant \tau \\ x, y \in L}}|\varphi(x)-\varphi(y)| .
$$

${ }^{1} a(n) \sim b(n)$ means that $C_{1} \leqslant \frac{a(n)}{b(n)} \leqslant C_{2}$, where $C_{1}$ and $C_{2}$ are positive constants independent of $n$. 
We consider the matrix $A^{n}=\left(a_{l j}\right)_{l, j=1}^{n}$ with the entries

$$
a_{l j}=-(2+i \mu)^{-1}\left(2 b_{i j}+4 i \mu \sum_{m=1}^{n} b_{l m} b_{m j}+\lambda(2+i \mu) c_{l j}+4 i \lambda \mu \sum_{m=1}^{n} e_{l m} c_{m j}\right)
$$

where

$$
\begin{aligned}
& b_{l l}=c_{l l}=e_{l l}=0 \quad \text { as } \quad l=\overline{1, n}, \\
& b_{l j}=\frac{(b-a)}{n} \frac{\partial \Phi\left(x\left(\tau_{l}\right), x\left(\tau_{j}\right)\right)}{\partial \vec{n}\left(x\left(\tau_{l}\right)\right)} \sqrt{\left(x_{1}^{\prime}\left(\tau_{j}\right)\right)^{2}+\left(x_{2}^{\prime}\left(\tau_{j}\right)\right)^{2}} \quad \text { as } \quad l, j=\overline{1, n} \quad \text { and } \quad l \neq j, \\
& c_{l j}=\frac{(b-a)}{n} \Phi\left(x\left(\tau_{l}\right), x\left(\tau_{j}\right)\right) \sqrt{\left(x_{1}^{\prime}\left(\tau_{j}\right)\right)^{2}+\left(x_{2}^{\prime}\left(\tau_{j}\right)\right)^{2}} \quad \text { as } \quad l, j=\overline{1, n} \quad \text { and } \quad l \neq j, \\
& e_{l j}=\frac{(b-a)}{n} \frac{\partial \Phi\left(x\left(\tau_{l}\right), x\left(\tau_{j}\right)\right)}{\partial \vec{n}\left(x\left(\tau_{j}\right)\right)} \sqrt{\left(x_{1}^{\prime}\left(\tau_{j}\right)\right)^{2}+\left(x_{2}^{\prime}\left(\tau_{j}\right)\right)^{2}} \quad \text { as } \quad l, j=\overline{1, n} \quad \text { and } \quad l \neq j .
\end{aligned}
$$

In what follows, by $M$ we denote inessential positive constants being different in various inequality.

Theorem 2.1. The expression

$$
(A \rho)^{n}\left(x\left(\tau_{l}\right)\right)=\sum_{j=1}^{n} a_{l j} \rho\left(x\left(\tau_{j}\right)\right),
$$

constructed by means of the nodes $x\left(\tau_{l}\right), l=\overline{1, n}$, is a quadrature formula for $(A \rho)(x)$, and the following estimate holds:

$$
\max _{l=\overline{1, n}}\left|(A \rho)\left(x\left(\tau_{l}\right)\right)-(A \rho)^{n}\left(x\left(\tau_{l}\right)\right)\right| \leqslant M\left[\omega\left(\rho, \frac{1}{n}\right)+\|\rho\|_{\infty} \frac{\ln n}{n}\right]
$$

where $\|\rho\|_{\infty}=\max _{x \in L}|\rho(x)|$.

Proof. It was proved in [8] that the expressions

$$
S_{n}\left(x\left(\tau_{k}\right)\right)=\frac{2(b-a)}{n} \sum_{\substack{j=1 \\ j \neq k}}^{n} \Phi\left(x\left(\tau_{k}\right), x\left(\tau_{j}\right)\right) \sqrt{\left(x_{1}^{\prime}\left(\tau_{j}\right)\right)^{2}+\left(x_{2}^{\prime}\left(\tau_{j}\right)\right)^{2}} \rho\left(x\left(\tau_{j}\right)\right)
$$

and

$$
\tilde{K}_{n}\left(x\left(\tau_{k}\right)\right)=\frac{2(b-a)}{n} \sum_{\substack{j=1 \\ j \neq k}}^{n} \frac{\partial \Phi\left(x\left(\tau_{k}\right), x\left(\tau_{j}\right)\right)}{\partial \vec{n}\left(x\left(\tau_{k}\right)\right)} \sqrt{\left(x_{1}^{\prime}\left(\tau_{j}\right)\right)^{2}+\left(x_{2}^{\prime}\left(\tau_{j}\right)\right)^{2}} \rho\left(x\left(\tau_{j}\right)\right),
$$

constructed by means of nodes $x\left(\tau_{k}\right), k=\overline{1, n}$, are quadrature formulae for the integrals $(S \rho)(x)$ and $(\tilde{K} \rho)(x)$, respectively, and

$$
\begin{aligned}
& \max _{k=1, n}\left|(S \rho)\left(x\left(\tau_{k}\right)\right)-S_{n}\left(x\left(\tau_{k}\right)\right)\right| \leqslant M\left(\omega\left(\rho, \frac{1}{n}\right)+\|\rho\|_{\infty} \frac{\ln n}{n}\right), \\
& \max _{k=1, n}\left|(\tilde{K} \rho)\left(x\left(\tau_{k}\right)\right)-\tilde{K}_{n}\left(x\left(\tau_{k}\right)\right)\right| \leqslant M\left(\omega\left(\rho, \frac{1}{n}\right)+\|\rho\|_{\infty} \frac{\ln n}{n}\right) .
\end{aligned}
$$

Now we are going to construct a quadrature formula for the integral $(Q \rho)(x)$. The expression

$$
(Q \rho)^{n}\left(x\left(\tau_{l}\right)\right)=\sum_{j=1}^{n}\left(\sum_{m=1}^{n} e_{l m} c_{m j}\right) \rho\left(x\left(\tau_{j}\right)\right)
$$


constructed by means of nodes $x\left(\tau_{l}\right), l=\overline{1, n}$, is a quadrature formula for the integral $(Q \rho)(x)$. Let us estimate the error of quadrature formula (2.2). It is obvious that

$$
\begin{aligned}
& (Q \rho)\left(x\left(\tau_{l}\right)\right)-(Q \rho)^{n}\left(x\left(\tau_{l}\right)\right)=(Q \rho)\left(x\left(\tau_{l}\right)\right)-\sum_{j=1}^{n}\left(e_{l j} \sum_{m=1}^{n} c_{j m} \rho\left(x\left(\tau_{m}\right)\right)\right) \\
& =\int_{L} \frac{\partial \Phi\left(x\left(\tau_{l}\right), y\right)}{\partial \vec{n}(y)}\left(\int_{L} \Phi(y, t) \rho(t) d L_{t}\right) d L_{y}-\frac{b-a}{n} \\
& \cdot \sum_{\substack{j=1 \\
j \neq l}}^{n}\left(\frac{\partial \Phi\left(x\left(\tau_{l}\right), x\left(\tau_{j}\right)\right)}{\partial \vec{n}\left(x\left(\tau_{j}\right)\right)} \operatorname{mes} L_{j} \sum_{\substack{m=1 \\
m \neq j}}^{n} \Phi\left(x\left(\tau_{j}\right), x\left(\tau_{m}\right)\right) \sqrt{\left(x_{1}^{\prime}\left(\tau_{m}\right)\right)^{2}+\left(x_{2}^{\prime}\left(\tau_{m}\right)\right)^{2}} \rho\left(x\left(\tau_{m}\right)\right)\right) \\
& +\sum_{\substack{j=1 \\
j \neq l}}^{n}\left(\frac{\partial \Phi\left(x\left(\tau_{l}\right), x\left(\tau_{j}\right)\right)}{\partial \vec{n}\left(x\left(\tau_{j}\right)\right)}\left(\operatorname{mes} L_{j}-\frac{b-a}{n} \sqrt{\left(x_{1}^{\prime}\left(\tau_{j}\right)\right)^{2}+\left(x_{2}^{\prime}\left(\tau_{j}\right)\right)^{2}}\right)\right. \\
& \left.\cdot \frac{b-a}{n} \sum_{\substack{m=1 \\
m \neq j}}^{n} \Phi\left(x\left(\tau_{j}\right), x\left(\tau_{m}\right)\right) \sqrt{\left(x_{1}^{\prime}\left(\tau_{m}\right)\right)^{2}+\left(x_{2}^{\prime}\left(\tau_{m}\right)\right)^{2}} \rho\left(x\left(\tau_{m}\right)\right)\right) \\
& =\int_{L_{l}} \frac{\partial \Phi\left(x\left(\tau_{l}\right), y\right)}{\partial \vec{n}(y)}\left(\int_{L} \Phi(y, t) \rho(t) d L_{t}\right) d L_{y} \\
& +\sum_{\substack{j=1 \\
j \neq l}}^{n} \int_{L_{j}} \frac{\partial \Phi\left(x\left(\tau_{l}\right), y\right)}{\partial \vec{n}(y)}\left(\int_{L} \Phi(y, t) \rho(t) d L_{t}-\int_{L} \Phi\left(x\left(\tau_{j}\right), t\right) \rho(t) d L_{t}\right) d L_{y} \\
& +\sum_{\substack{j=1 \\
j \neq l}}^{n}\left[\int_{L_{j}}\left(\frac{\partial \Phi\left(x\left(\tau_{l}\right), y\right)}{\partial \vec{n}(y)}-\frac{\partial \Phi\left(x\left(\tau_{l}\right), x\left(\tau_{j}\right)\right)}{\partial \vec{n}\left(x\left(\tau_{j}\right)\right)}\right) \int_{L} \Phi\left(x\left(\tau_{j}\right), t\right) \rho(t) d L_{t}\right] d L_{y} \\
& +\sum_{\substack{j=1 \\
j \neq l}}^{n} \frac{\partial \Phi\left(x\left(\tau_{l}\right), x\left(\tau_{j}\right)\right)}{\partial \vec{n}\left(x\left(\tau_{j}\right)\right)}\left(\int_{L} \Phi\left(x\left(\tau_{j}\right), t\right) \rho(t) d S_{t}\right. \\
& \left.-\frac{b-a}{n} \sum_{\substack{m=1 \\
m \neq j}}^{n} \Phi\left(x\left(\tau_{j}\right), x\left(\tau_{m}\right)\right) \sqrt{\left(x_{1}^{\prime}\left(\tau_{m}\right)\right)^{2}+\left(x_{2}^{\prime}\left(\tau_{m}\right)\right)^{2}} \rho\left(x\left(\tau_{m}\right)\right)\right) \operatorname{mes} L_{j} \\
& +\sum_{\substack{j=1 \\
j \neq l}}^{n}\left(\frac{\partial \Phi\left(x\left(\tau_{l}\right), x\left(\tau_{j}\right)\right)}{\partial \vec{n}\left(x\left(\tau_{j}\right)\right)}\left(\operatorname{mes} L_{j}-\frac{b-a}{n} \sqrt{\left(x_{1}^{\prime}\left(\tau_{j}\right)\right)^{2}+\left(x_{2}^{\prime}\left(\tau_{j}\right)\right)^{2}}\right)\right. \\
& \left.\cdot \frac{b-a}{n} \sum_{\substack{m=1 \\
m \neq j}}^{n} \Phi\left(x\left(\tau_{j}\right), x\left(\tau_{m}\right)\right) \sqrt{\left(x_{1}^{\prime}\left(\tau_{m}\right)\right)^{2}+\left(x_{2}^{\prime}\left(\tau_{m}\right)\right)^{2}} \rho\left(x\left(\tau_{m}\right)\right)\right) .
\end{aligned}
$$

The terms in the right hand side of the latter inequality is denoted by $Q_{1}(n), Q_{2}(n), Q_{3}(n)$, $Q_{4}(n)$ and $Q_{5}(n)$, respectively. 
Taking into consideration that the curve $L$ is twice continuously differentiable, we have (see [7, Ch. V]):

$$
|(x-y, \vec{n}(y))|=|x-y||\cos \alpha(x-y, \vec{n}(y))| \leqslant M|x-y|^{2}, \quad x, y \in L,
$$

where by $\alpha(x-y, \vec{n}(y))$ we denote an angle between the vectors $x-y$ and $\vec{n}(y)$. Then

$$
\left|\frac{\partial \Phi(x, y)}{\partial \vec{n}(y)}\right|=\frac{1}{2 \pi} \frac{|(x-y, \vec{n}(y))|}{|x-y|^{2}} \leqslant M, \quad x, y \in L, \quad x \neq y .
$$

Since the operator $S$ is bounded as acting from the space $C(L)$ into the space $C(L)$, then

$$
\left|Q_{1}(n)\right| \leqslant\left\|\int_{L} \Phi(y, t) \rho(t) d L_{t}\right\|_{\infty} \int_{L_{l}}\left|\frac{\partial \Phi\left(x\left(\tau_{l}\right), y\right)}{\partial \vec{n}(y)}\right| d L_{y} \leqslant M\|\rho\|_{\infty} \int_{L_{l}} d L_{y} \leqslant M\|\rho\|_{\infty} R(n) .
$$

Moreover, taking into consideration inequality

$$
\omega(S \rho, h) \leqslant M\|\rho\|_{\infty} h|\ln h|,
$$

see [10, Thm. 2.12], we obtain that for each $y \in L_{j}$

$$
\left|\int_{L} \Phi(y, t) \rho(t) d L_{t}-\int_{L} \Phi\left(x\left(\tau_{j}\right), t\right) \rho(t) d L_{t}\right| \leqslant M\|\rho\|_{\infty} R(n)|\ln R(n)|,
$$

and therefore,

$$
\left|Q_{2}(n)\right| \leqslant M\|\rho\|_{\infty} R(n)|\ln R(n)| \int_{L}\left|\frac{\partial \Phi\left(x\left(\tau_{l}\right), y\right)}{\partial \vec{n}(y)}\right| d L_{y} \leqslant M\|\rho\|_{\infty} R(n)|\ln R(n)| .
$$

Bearing in mind Lemma 2.1, we get that for each $y \in L_{j}$ and for all $l, j \in\{1,2, \ldots, n\}, j \neq l$,

$$
\begin{aligned}
\left|\frac{\partial \Phi\left(x\left(\tau_{l}\right), y\right)}{\partial \vec{n}(y)}-\frac{\partial \Phi\left(x\left(\tau_{l}\right), x\left(\tau_{j}\right)\right)}{\partial \vec{n}\left(x\left(\tau_{j}\right)\right)}\right| & =\mid \frac{\left(x\left(\tau_{l}\right)-y, \vec{n}(y)\right)\left(\left|x\left(\tau_{l}\right)-x\left(\tau_{j}\right)\right|^{2}-\left|x\left(\tau_{l}\right)-y\right|^{2}\right)}{\left|x\left(\tau_{l}\right)-y\right|^{2}\left|x\left(\tau_{l}\right)-x\left(\tau_{j}\right)\right|^{2}} \\
& -\frac{\left(x\left(\tau_{l}\right)-y, \vec{n}(y)-\vec{n}\left(x\left(\tau_{j}\right)\right)+\left(x\left(\tau_{j}\right)-y, \vec{n}\left(x\left(\tau_{j}\right)\right)\right)\right.}{\left|x\left(\tau_{l}\right)-x\left(\tau_{j}\right)\right|^{2}} \mid \\
& \leqslant M \frac{\left|x\left(\tau_{j}\right)-y\right|}{\left|x\left(\tau_{l}\right)-y\right|} .
\end{aligned}
$$

Then

$$
\begin{aligned}
\left|Q_{3}(n)\right| & \leqslant M\left\|\int_{L} \Phi(x, t) \rho(t) d L_{t}\right\| \sum_{\substack{j=1 \\
j \neq l}}^{n} \int_{L_{j}}\left|\frac{\partial \Phi\left(x\left(\tau_{l}\right), y\right)}{\partial \vec{n}(y)}-\frac{\partial \Phi\left(x\left(\tau_{l}\right), x\left(\tau_{j}\right)\right)}{\partial \vec{n}\left(x\left(\tau_{j}\right)\right)}\right| d L_{y} \\
& \leqslant M\|\rho\|_{\infty} R(n) \int_{L \backslash L_{l}} \frac{d L_{y}}{\left|x\left(\tau_{l}\right)-y\right|} \leqslant M\|\rho\|_{\infty} R(n)|\ln R(n)| .
\end{aligned}
$$

Taking into consideration inequality (2.4) and the estimate for error term in the quadrature formulae for the logarithmic single layer potential, we find:

$$
\left|Q_{4}(n)\right| \leqslant M\left[\|\rho\|_{\infty} R(n)|\ln R(n)|+\omega(\rho, R(n))\right] .
$$

In view of the inequality

$$
\left|\sqrt{\left(x_{1}^{\prime}(t)\right)^{2}+\left(x_{2}^{\prime}(t)\right)^{2}}-\sqrt{\left(x_{1}^{\prime}\left(\tau_{j}\right)\right)^{2}+\left(x_{2}^{\prime}\left(\tau_{j}\right)\right)^{2}}\right| \leqslant M R(n), \quad t \in\left[t_{j-1}, t_{j}\right],
$$


we obtain:

$$
\begin{aligned}
\left|1-\frac{\frac{b-a}{n} \sqrt{\left(x_{1}^{\prime}\left(\tau_{j}\right)\right)^{2}+\left(x_{2}^{\prime}\left(\tau_{j}\right)\right)^{2}}}{\operatorname{mes} L_{j}}\right| & =\frac{\left|\int_{t_{j-1}}^{t_{j}}\left(\sqrt{\left(x_{1}^{\prime}(t)\right)^{2}+\left(x_{2}^{\prime}(t)\right)^{2}}-\sqrt{\left(x_{1}^{\prime}\left(\tau_{j}\right)\right)^{2}+\left(x_{2}^{\prime}\left(\tau_{j}\right)\right)^{2}}\right) d t\right|}{\int_{t_{j-1}}^{t_{j}} \sqrt{\left(x_{1}^{\prime}(t)\right)^{2}+\left(x_{2}^{\prime}(t)\right)^{2}} d t} \\
& \leqslant M \frac{\frac{b-a}{n} R(n)}{\frac{b-a}{n} m_{1}} \leqslant M R(n),
\end{aligned}
$$

where

$$
m_{1}=\min _{t \in[a, b]} \sqrt{\left(x_{1}^{\prime}(t)\right)^{2}+\left(x_{2}^{\prime}(t)\right)^{2}}>0
$$

see [6, Ch. VI]. Moreover, it is obvious that

$$
\frac{\frac{b-a}{n} \sqrt{\left(x_{1}^{\prime}\left(\tau_{j}\right)\right)^{2}+\left(x_{2}^{\prime}\left(\tau_{j}\right)\right)^{2}}}{\operatorname{mes} L_{j}} \leqslant M .
$$

Then, owing to Lemma 2.1, we obtain:

$$
\begin{aligned}
\left|Q_{5}(n)\right|= & \mid \sum_{\substack{j=1 \\
j \neq l}}^{n}\left(\frac{\partial \Phi\left(x\left(\tau_{l}\right), x\left(\tau_{j}\right)\right)}{\partial \vec{n}\left(x\left(\tau_{j}\right)\right)} \operatorname{mes} L_{j}\left(1-\frac{\frac{b-a}{n} \sqrt{\left(x_{1}^{\prime}\left(\tau_{j}\right)\right)^{2}+\left(x_{2}^{\prime}\left(\tau_{j}\right)\right)^{2}}}{\operatorname{mes} L_{j}}\right)\right. \\
& \left.\cdot \sum_{\substack{m=1 \\
m \neq j}}^{n} \Phi\left(x\left(\tau_{j}\right), x\left(\tau_{m}\right)\right) \operatorname{mes} L_{m} \frac{\frac{b-a}{n} \sqrt{\left(x_{1}^{\prime}\left(\tau_{m}\right)\right)^{2}+\left(x_{2}^{\prime}\left(\tau_{m}\right)\right)^{2}}}{\operatorname{mes} L_{m}} \rho\left(x\left(\tau_{m}\right)\right)\right) \mid \\
\leqslant & M R(n)\|\rho\|_{\infty} \int_{L \backslash L_{l}}\left|\frac{\partial \Phi\left(x\left(\tau_{l}\right), y\right)}{\partial \vec{n}(y)}\right| d y \int_{L \backslash L_{j}}\left|\Phi\left(x\left(\tau_{j}\right), t\right)\right| d t \leqslant M R(n)\|\rho\|_{\infty} .
\end{aligned}
$$

Thus, summing up the obtained estimates for the expressions $Q_{1}(n), Q_{2}(n), Q_{3}(n), Q_{4}(n)$ and $Q_{5}(n)$, we find:

$$
\max _{l=\overline{1, n}}\left|(Q \rho)\left(x\left(\tau_{l}\right)\right)-(Q \rho)^{n}\left(x\left(\tau_{l}\right)\right)\right| \leqslant M\left[\|\rho\|_{\infty} R(n)|\ln R(n)|+\omega(\rho, R(n))\right] .
$$

In the same way one can show that the expression

$$
(R \rho)^{n}\left(x\left(\tau_{l}\right)\right)=\sum_{j=1}^{n}\left(\sum_{m=1}^{n} b_{l m} b_{m j}\right) \rho\left(x\left(\tau_{j}\right)\right)
$$

constructed by means of nodes $x\left(\tau_{l}\right), l=\overline{1, n}$, is a quadrature formula for integral $(R \rho)(x)$, and

$$
\max _{l=\overline{1, n}}\left|(R \rho)\left(x\left(\tau_{l}\right)\right)-(R \rho)^{n}\left(x\left(\tau_{l}\right)\right)\right| \leqslant M\left[\|\rho\|_{\infty} R(n)|\ln R(n)|+\omega(\rho, R(n))\right] .
$$

As a result, taking into consideration the constructed quadrature formulae for the integrals $(S \rho)(x),(\tilde{K} \rho)(x),(Q \rho)(x),(R \rho)(x)$ and the estimates for their errors, we obtain that expression (2.1) constructed by means of nodes $x\left(\tau_{l}\right), l=\overline{1, n}$, is a quadrature formula for $(A \rho)(x)$, and

$$
\max _{l=1, n}\left|(A \rho)\left(x\left(\tau_{l}\right)\right)-(A \rho)^{n}\left(x\left(\tau_{l}\right)\right)\right| \leqslant M\left[\|\rho\|_{\infty} R(n)|\ln R(n)|+\omega(\rho, R(n))\right] .
$$

Then, in view of the relation $R(n) \sim \frac{1}{n}$, we complete the proof. 


\section{JUSTIFICATION OF COLLOCATION METHOD}

Let $\mathbb{C}^{n}$ be the space of $n$-dimensional vectors $z^{n}=\left(z_{1}^{n}, z_{2}^{n}, \ldots, z_{n}^{n}\right)^{\mathrm{T}}, z_{l}^{n} \in \mathbb{C}, l=\overline{1, n}$, with the norm $\left\|z^{n}\right\|=\max _{l=\overline{1, n}}\left|z_{l}^{n}\right|$, where the writing " $a^{\mathrm{T}}$ " denotes the transposition of a vector $a$. Employing quadrature formula (2.1), we replace integral equation (1.1) by a system of algebraic equations with respect to approximate values $z_{l}^{n}$ of $\rho\left(x\left(\tau_{l}\right)\right), l=\overline{1, n}$, which we write as

$$
\left(I^{n}+A^{n}\right) z^{n}=\varphi^{n}
$$

where $I^{n}$ is the identity mapping on the space $\mathbb{C}^{n}$,

$$
\varphi^{n}=-4(2+i \mu)^{-1} p^{n} f
$$

and $p^{n}: C(L) \rightarrow \mathbb{C}^{n}$ is a linear bounded operator defined by the formula

$$
p^{n} f=\left(f\left(x\left(\tau_{1}\right)\right), f\left(x\left(\tau_{2}\right)\right), \ldots, f\left(x\left(\tau_{n}\right)\right)\right)^{\mathrm{T}}
$$

and called a simple drift operator.

We obtain the justification by Vainikko convergence theorem for linear operator equations 11. In order to formulate it, in terms of notations in work [11, we provide needed definitions and statements.

Definition 3.1. [11]. Let $E$ and $E_{n}$ be Banach spaces. The system $Q=\left\{q^{n}\right\}$ of operators $q^{n}: E \rightarrow E_{n}$ is called connecting for $E$ and $E_{n}$ if for all $\varphi, \varphi^{\prime} \in E$ and $a, a^{\prime} \in \mathbb{C}$ we have

$$
\begin{aligned}
& \left\|q^{n} \varphi\right\| \rightarrow\|\varphi\|_{\infty} \quad \text { as } \quad n \rightarrow \infty ; \\
& \left\|q^{n}\left(a \varphi+a^{\prime} \varphi^{\prime}\right)-\left(a q^{n} \varphi+a^{\prime} q^{n} \varphi^{\prime}\right)\right\| \rightarrow 0 \quad \text { as } \quad n \rightarrow \infty .
\end{aligned}
$$

Definition 3.2 ([11, Def. 1.1]). A sequence $\left\{\varphi_{n}\right\}$ of elements $\varphi_{n} \in E_{n} Q$-converges to $\varphi \in E$ if $\left\|\varphi_{n}-q^{n} \varphi\right\| \rightarrow 0$ as $n \rightarrow \infty$. We shall write this as $\varphi_{n} \stackrel{Q}{\rightarrow} \varphi$.

Definition 3.3 ([11, Def. 1.2]). A sequence $\left\{\varphi_{n}\right\}$ of elements $\varphi_{n} \in E_{n}$ is Q-compact if each its subsequence $\left\{\varphi_{n_{m}}\right\}$ contains a $Q$-converging subsequence $\left\{\varphi_{n_{m_{k}}}\right\}$.

Proposition 3.1 ([11, Prop. 1.1]). Let a system $Q=\left\{q^{n}\right\}$ of linear bounded operators $q^{n}$ : $E \rightarrow E_{n}$ be connecting for $E$ and $E_{n}$. Then the following conditions are equivalent:

1. a sequence $\left\{\varphi_{n}\right\}$ is $Q$-compact and the set of its Q-limiting points is compact in $E$;

2. there exists a relatively compact sequence $\left\{\varphi^{(n)}\right\} \subset E$ such that $\left\|\varphi_{n}-q^{n} \varphi^{(n)}\right\| \rightarrow 0$ as $n \rightarrow \infty$.

Definition 3.4 ([11, Def. 2.1]). A sequence of operators $A^{n}: E_{n} \rightarrow E_{n} Q Q$-converges to an operator $A: E \rightarrow E$, if for each $Q$-converging sequence $\left\{\varphi_{n}\right\}$ we have $\varphi_{n} \stackrel{Q}{\rightarrow} \varphi \Rightarrow A^{n} \varphi_{n} \stackrel{Q}{\rightarrow} A \varphi$. We shall write this as $A^{n} \stackrel{Q Q}{\rightarrow} A$.

Definition 3.5 ([11, Def. 3.3]). . A sequence of operators $A^{n} \in L\left(E_{n}, E_{n}\right)$ compactly converges to an operator $A \in L(E, E)$ if $A^{n} \stackrel{Q Q}{\rightarrow} A$ and the following compactness condition holds:

$$
\varphi_{n} \in E_{n}, \quad\left\|\varphi_{n}\right\| \leqslant M \quad \Rightarrow \quad\left\{A^{n} \varphi_{n}\right\} \quad \text { is Q-compact. }
$$

Theorem 3.1. [11, Thm. 4.2] Assume that the following conditions hold:

1. $\operatorname{Ker}(I+A)=\{0\}$, where $I$ is the identity mapping in the space $E$;

2. the operators $I^{n}+A^{n}$ are Fredholm with a zero index;

3. $\psi_{n} \stackrel{Q}{\rightarrow} \psi, \psi_{n} \in E_{n}, \psi \in E$;

4. $A^{n} \rightarrow A$ is compact. 
Then the equation

$$
(I+A) \varphi=\psi
$$

possesses a unique solution $\tilde{\varphi} \in E$, the equation

$$
\left(I^{n}+A^{n}\right) \varphi_{n}=\psi_{n}
$$

also possesses a unique solution $\tilde{\varphi}_{n} \in E_{n}$, and $\tilde{\varphi}_{n} \stackrel{Q}{\rightarrow} \tilde{\varphi}$ with the estimates

$$
c_{1}\left\|\left(I^{n}+A^{n}\right) q^{n} \tilde{\varphi}-\psi_{n}\right\| \leqslant\left\|\tilde{\varphi}_{n}-q^{n} \tilde{\varphi}\right\| \leqslant c_{2}\left\|\left(I^{n}+A^{n}\right) q^{n} \tilde{\varphi}-\psi_{n}\right\|,
$$

where

$$
c_{1}=\frac{1}{\sup _{n}\left\|I^{n}+A^{n}\right\|}>0, \quad c_{2}=\sup _{n}\left\|\left(I^{n}+A^{n}\right)^{-1}\right\|<+\infty .
$$

Theorem 3.2. Equations (1.1) and (3.1) possess unique solutions $\rho_{*} \in C(S)$ and $z_{*}^{n} \in \mathbb{C}^{n}$, respectively, and $\left\|z_{*}^{n}-p^{n} \rho_{*}\right\| \rightarrow 0$ as $n \rightarrow \infty$ with the estimate

$$
\left\|z_{*}^{n}-p^{n} \rho_{*}\right\| \leqslant M\left[\omega\left(f, \frac{1}{n}\right)+\|f\|_{\infty} \frac{\ln n}{n}\right] .
$$

Proof. Since equation (1.1) is uniquely solvable, then $\operatorname{Ker}(I+A)=\{0\}$. It is obvious that the operators $I^{n}+A^{n}$ are Fredholm with the zero index and the operators $p^{n}: C(L) \rightarrow \mathbb{C}^{n}$ are linear and bounded. Taking into consideration the way of partitioning the curve $L$ into elementary parts, we obtain that for each $g \in C(L)$

$$
\lim _{n \rightarrow \infty}\left\|p^{n} g\right\|=\lim _{n \rightarrow \infty} \max _{l=1, n}\left|g\left(x\left(\tau_{l}\right)\right)\right|=\max _{x \in L}|g(x)|=\|g\|_{\infty} .
$$

Therefore, the system of simple drift operators $P=\left\{p^{n}\right\}$ is connecting for the spaces $C(L)$ and $\mathbb{C}^{n}$. Then $\varphi^{n} \stackrel{P}{\rightarrow} \varphi$ and by Theorem 2.1 we obtain that $I^{n}+A^{n} \stackrel{P P}{\rightarrow} I+A$. By Definition 3.5 . it remains to confirm the compactness condition. In view of Proposition 3.1, it is equivalent to the following condition: for each $\left\{z^{n}\right\}, z^{n} \in \mathbb{C}^{n},\left\|z^{n}\right\| \leqslant M$, there exists a relatively compact sequence $\left\{A_{n} z^{n}\right\} \subset C(L)$ such that

$$
\left\|A^{n} z^{n}-p^{n}\left(A_{n} z^{n}\right)\right\| \rightarrow 0 \quad \text { as } \quad n \rightarrow \infty .
$$

As $\left\{A_{n} z^{n}\right\}$, we choose a sequence

$$
\begin{aligned}
\left(A_{n} z^{n}\right)(x)= & -(2+i \mu)^{-1}\left(2\left(\tilde{K}_{n} z^{n}\right)(x)+4 i \mu\left(R_{n} z^{n}\right)(x)\right. \\
& \left.+\lambda(2+i \mu)\left(S_{n} z^{n}\right)(x)+4 i \lambda \mu\left(Q_{n} z^{n}\right)(x)\right),
\end{aligned}
$$

where

$$
\begin{aligned}
& \left(S_{n} z^{n}\right)(x)=2 \sum_{j=1}^{n} z_{j}^{n} \int_{L_{j}} \Phi(x, y) d L_{y}, \quad x \in L, \\
& \left(\tilde{K}_{n} z^{n}\right)(x)=2 \sum_{j=1}^{n} z_{j}^{n} \int_{L_{j}} \frac{\partial \Phi(x, y)}{\partial \vec{n}(x)} d L_{y}, \quad x \in L, \\
& \left(R_{n} z^{n}\right)(x)=\sum_{j=1}^{n} z_{j}^{n} \int_{L} \frac{\partial \Phi(x, y)}{\partial \vec{n}(x)}\left(\int_{L_{j}} \frac{\partial \Phi(y, t)}{\partial \vec{n}(y)} d L_{t}\right) d L_{y}, \quad x \in L, \\
& \left(Q_{n} z^{n}\right)(x)=\sum_{j=1}^{n} z_{j}^{n} \int_{L} \frac{\partial \Phi(x, y)}{\partial \vec{n}(y)}\left(\int_{L_{j}} \Phi(y, t) d L_{t}\right) d L_{y}, \quad x \in L .
\end{aligned}
$$


Let $L_{d}(x)=\{y \in L:|y-x|<d\}$. We take arbitrary points $x^{\prime}, x^{\prime \prime} \in L$ such that

$$
\left|x^{\prime}-x^{\prime \prime}\right|=\delta<\frac{\min \{1, d\}}{2} \text {. }
$$

Since

$$
\left|\sum_{j=1}^{n} z_{j}^{n} \int_{L_{j}} \Phi(y, t) d L_{t}\right| \leqslant\left\|z^{n}\right\| \int_{L}|\Phi(y, t)| d L_{t} \leqslant M\left\|z^{n}\right\|,
$$

then

$$
\begin{aligned}
\mid\left(Q_{n} z^{n}\right)\left(x^{\prime}\right)- & \left(Q_{n} z^{n}\right)\left(x^{\prime \prime}\right) \mid \\
\leqslant & M\left\|z^{n}\right\| \int\left|\frac{\partial \Phi\left(x^{\prime}, y\right)}{\partial \vec{n}(y)}-\frac{\partial \Phi\left(x^{\prime \prime}, y\right)}{\partial \vec{n}(y)}\right| d L_{y} \\
\leqslant & M\left\|z^{n}\right\| \int_{L_{\frac{\delta}{2}}\left(x^{\prime}\right)}\left|\frac{\partial \Phi\left(x^{\prime}, y\right)}{\partial \vec{n}(y)}\right| d L_{y}+M\left\|z^{n}\right\| \int_{L_{\frac{\delta}{2}}\left(x^{\prime \prime}\right)}\left|\frac{\partial \Phi\left(x^{\prime \prime}, y\right)}{\partial \vec{n}(y)}\right| d L_{y} \\
& +M\left\|z^{n}\right\| \int_{L_{\frac{\delta}{2}}\left(x^{\prime}\right)}\left|\frac{\partial \Phi\left(x^{\prime \prime}, y\right)}{\partial \vec{n}(y)}\right| d L_{y}+M\left\|z^{n}\right\| \int_{\frac{\delta}{2}}\left|\frac{\partial \Phi\left(x^{\prime \prime}\right)}{\partial \vec{n}(y)}\right| d L_{y} \\
& +M\left\|z^{n}\right\|\left|\frac{\partial \Phi\left(x^{\prime}, y\right)}{\partial \vec{n}(y)}-\frac{\partial \Phi\left(x^{\prime \prime}, y\right)}{\partial \vec{n}(y)}\right| d L_{y} \\
& +M\left\|z^{n}\right\| \underset{L_{d}\left(x^{\prime}\right) \backslash\left(L_{\frac{\delta}{2}}\left(x^{\prime}\right) \cup L_{\frac{\delta}{2}}\left(x^{\prime \prime}\right)\right)}{\int\left|\frac{\partial \Phi\left(x^{\prime}, y\right)}{\partial \vec{n}(y)}-\frac{\partial \Phi\left(x^{\prime \prime}, y\right)}{\partial \vec{n}(y)}\right| d L_{y} .}
\end{aligned}
$$

Taking into consideration inequality (2.4), we have:

$$
\begin{aligned}
& \int_{L_{\frac{\delta}{2}}\left(x^{\prime}\right)}\left|\frac{\partial \Phi\left(x^{\prime}, y\right)}{\partial \vec{n}(y)}\right| d L_{y}=\frac{1}{2 \pi} \int_{L_{\frac{\delta}{2}}\left(x^{\prime}\right)} \frac{\left|\left(x^{\prime}-y, \vec{n}(y)\right)\right|}{\left|x^{\prime}-y\right|^{2}} d L_{y} \leqslant M \int_{L_{\frac{\delta}{2}}\left(x^{\prime}\right)} d L_{y} \leqslant M \delta, \\
& \int_{L_{\frac{\delta}{2}}\left(x^{\prime \prime}\right)}\left|\frac{\partial \Phi\left(x^{\prime \prime}, y\right)}{\partial \vec{n}(y)}\right| d L_{y} \leqslant M \delta, \\
& \int_{L_{\frac{\delta}{2}}\left(x^{\prime}\right)}\left|\frac{\partial \Phi\left(x^{\prime \prime}, y\right)}{\partial \vec{n}(y)}\right| d L_{y}=\frac{1}{2 \pi} \int_{L_{\frac{\delta}{2}}\left(x^{\prime}\right)} \frac{\left|\left(x^{\prime \prime}-y, \vec{n}(y)\right)\right|}{\left|x^{\prime \prime}-y\right|^{2}} d L_{y} \leqslant M \int_{L_{\frac{\delta}{2}}\left(x^{\prime}\right)} d L_{y} \leqslant M \delta
\end{aligned}
$$

and

$$
\int_{L_{\frac{\delta}{2}}\left(x^{\prime \prime}\right)}\left|\frac{\partial \Phi\left(x^{\prime}, y\right)}{\partial \vec{n}(y)}\right| d L_{y} \leqslant M \delta
$$

Since

$$
\begin{aligned}
\frac{\partial \Phi\left(x^{\prime}, y\right)}{\partial \vec{n}(y)}-\frac{\partial \Phi\left(x^{\prime \prime}, y\right)}{\partial \vec{n}(y)} & =\frac{1}{2 \pi}\left(\frac{\left(x^{\prime}-y, \vec{n}(y)\right)}{\left|x^{\prime}-y\right|^{2}}-\frac{\left(x^{\prime \prime}-y, \vec{n}(y)\right)}{\left|x^{\prime \prime}-y\right|^{2}}\right) \\
& =\frac{\left(x^{\prime}-y, \vec{n}(y)\right)\left(\left|x^{\prime \prime}-y\right|-\left|x^{\prime}-y\right|\right)\left(\left|x^{\prime \prime}-y\right|+\left|x^{\prime}-y\right|\right)}{2 \pi\left|x^{\prime}-y\right|^{2}\left|x^{\prime \prime}-y\right|^{2}}
\end{aligned}
$$




$$
+\frac{\left(x^{\prime}-x^{\prime \prime}, \vec{n}(y)\right)}{2 \pi\left|x^{\prime \prime}-y\right|^{2}}
$$

and for each $y \in L_{d}\left(x^{\prime}\right) \backslash\left(L_{\frac{\delta}{2}}\left(x^{\prime}\right) \bigcup L_{\frac{\delta}{2}}\left(x^{\prime \prime}\right)\right)$

$$
\left|x^{\prime}-y\right| \leqslant\left|x^{\prime}-x^{\prime \prime}\right|+\left|x^{\prime \prime}-y\right| \leqslant 3\left|x^{\prime \prime}-y\right|, \quad\left|x^{\prime \prime}-y\right| \leqslant 3\left|x^{\prime}-y\right|,
$$

then, in view of inequality (2.3), we find:

$$
\left|\frac{\partial \Phi\left(x^{\prime}, y\right)}{\partial \vec{n}(y)}-\frac{\partial \Phi\left(x^{\prime \prime}, y\right)}{\partial \vec{n}(y)}\right| \leqslant \frac{M \delta}{\left|x^{\prime}-y\right|}, \quad y \in L_{d}\left(x^{\prime}\right) \backslash\left(L_{\frac{\delta}{2}}\left(x^{\prime}\right) \bigcup L_{\frac{\delta}{2}}\left(x^{\prime \prime}\right)\right) .
$$

Hence,

$$
\begin{gathered}
\int_{L_{d}\left(x^{\prime}\right) \backslash\left(L_{\frac{\delta}{2}}\left(x^{\prime}\right) \cup L_{\frac{\delta}{2}}\left(x^{\prime \prime}\right)\right)} \quad\left|\frac{\partial \Phi\left(x^{\prime}, y\right)}{\partial \vec{n}(y)}-\frac{\partial \Phi\left(x^{\prime \prime}, y\right)}{\partial \vec{n}(y)}\right| d L_{y} \\
\leqslant M \delta \int_{L_{d}\left(x^{\prime}\right) \backslash\left(L_{\frac{\delta}{2}}\left(x^{\prime}\right) \cup L_{\frac{\delta}{2}}\left(x^{\prime \prime}\right)\right)} \frac{d L_{y}}{\left|x^{\prime}-y\right|} \\
\leqslant M \delta \int_{\delta}^{d} \frac{d t}{t} \leqslant M \delta|\ln \delta| .
\end{gathered}
$$

Moreover, it is obvious that

$$
\int_{L \backslash L_{d}\left(x^{\prime}\right)}\left|\frac{\partial \Phi\left(x^{\prime}, y\right)}{\partial \vec{n}(y)}-\frac{\partial \Phi\left(x^{\prime \prime}, y\right)}{\partial \vec{n}(y)}\right| d L_{y} \leqslant M \delta .
$$

Summing up the above estimates, we find:

$$
\left|\left(Q_{n} z^{n}\right)\left(x^{\prime}\right)-\left(Q_{n} z^{n}\right)\left(x^{\prime \prime}\right)\right| \leqslant M\left\|z^{n}\right\| \delta|\ln \delta| .
$$

In the same way one can prove this estimate for the sequences $\left\{S_{n} z^{n}\right\},\left\{\tilde{K}_{n} z^{n}\right\}$ and $\left\{R_{n} z^{n}\right\}$. Then

$$
\left|\left(A_{n} z^{n}\right)\left(x^{\prime}\right)-\left(A_{n} z^{n}\right)\left(x^{\prime \prime}\right)\right| \leqslant M\left\|z^{n}\right\| \delta|\ln \delta|,
$$

and therefore, $\left\{A_{n} z^{n}\right\} \subset C(L)$.

By the inequality $\left\|z^{n}\right\| \leqslant M$ we find that the sequence $\left\{A_{n} z^{n}\right\}$ is uniformly bounded, while estimate (3.3) yields that this sequence is equicontinuous. Then by Arzelà-Ascoli we conclude on a relative compactness of the sequence $\left\{A_{n} z^{n}\right\}$. Taking into consideration the way of partitioning the curve $L$ into elementary parts and Lemma 2.1, it is easy to show that

$$
\left\|A^{n} z^{n}-p^{n}\left(A_{n} z^{n}\right)\right\| \rightarrow 0 \quad \text { as } \quad n \rightarrow \infty .
$$

Then, applying Theorem 3.1, we obtain that equations (1.1) and (3.1) have unique solutions $\rho_{*} \in C(S)$ and $z_{*}^{n} \in \mathbb{C}^{n}$, respectively, and

$$
c_{1} \delta_{n} \leqslant\left\|z_{*}^{n}-p^{n} \rho_{*}\right\| \leqslant c_{2} \delta_{n}
$$

where

$$
\begin{aligned}
c_{1} & =\frac{1}{\sup _{n}\left\|I^{n}+A^{n}\right\|}>0, \quad c_{2}=\sup _{n}\left\|\left(I^{n}+A^{n}\right)^{-1}\right\|<\infty, \\
\delta_{n} & =\left\|\left(I^{n}+A^{n}\right)\left(p^{n} \rho_{*}\right)-\varphi^{n}\right\| .
\end{aligned}
$$


Applying Theorem 2.1, we obtain:

$$
\begin{aligned}
\delta_{n} & =\left\|p^{n} \rho_{*}+A^{n}\left(p^{n} \rho_{*}\right)-p^{n}\left(\rho_{*}+A \rho_{*}\right)\right\|=\left\|A^{n}\left(p^{n} \rho_{*}\right)-p^{n}\left(A \rho_{*}\right)\right\| \\
& =\max _{l=1, n}\left|\sum_{j=1}^{n} a_{l j} \rho_{*}\left(x\left(\tau_{j}\right)\right)-\left(A \rho_{*}\right)\left(x\left(\tau_{l}\right)\right)\right| \leqslant M\left[\|\rho\|_{\infty} \frac{\ln n}{n}+\omega\left(\rho, \frac{1}{n}\right)\right] .
\end{aligned}
$$

Since the operator $A$ is a weakly singular integral operator, it is compact in $C(L)$ [10, Thm. 2.6]. Moreover, the unique solvability of integral equation (1.1) obviously implies that the operator $I+A$ is injective and hence, the inverse operator $(I+A)^{-1}$ is bounded [10, Thm. 1.16]. Then

$$
\left\|\rho_{*}\right\|_{\infty}=\left\|(I+A)^{-1} \varphi\right\|_{\infty} \leqslant\left\|(I+A)^{-1}\right\|\|\varphi\|_{\infty} \leqslant M\|f\|_{\infty} .
$$

Moreover, proceeding as in the proof of inequality (3.2), one can show that

$$
\omega\left(R \rho_{*}, h\right) \leqslant M\left\|\rho_{*}\right\|_{\infty} h|\ln h|
$$

and

$$
\omega\left(Q \rho_{*}, h\right) \leqslant M\left\|\rho_{*}\right\|_{\infty} h|\ln h| .
$$

Then, taking into consideration inequalities

$$
\omega\left(S \rho_{*}, h\right) \leqslant M\left\|\rho_{*}\right\|_{\infty} h|\ln h|
$$

and

$$
\omega\left(K \rho_{*}, h\right) \leqslant M\left\|\rho_{*}\right\|_{\infty} h|\ln h|,
$$

see [10, Thms. 2.12, 2.16], we find:

$$
\omega\left(A \rho_{*}, \frac{1}{n}\right) \leqslant M\left\|\rho_{*}\right\|_{\infty} \frac{\ln n}{n} \leqslant M\|f\|_{\infty} \frac{\ln n}{n} .
$$

Hence,

$$
\begin{aligned}
\omega\left(\rho_{*}, \frac{1}{n}\right) & =\omega\left(\varphi-A \rho_{*}, \frac{1}{n}\right) \leqslant \omega\left(\varphi, \frac{1}{n}\right)+\omega\left(A \rho_{*}, \frac{1}{n}\right) \\
& \leqslant \omega\left(\varphi, \frac{1}{n}\right)+M\|f\|_{\infty} \frac{\ln n}{n} \leqslant M\left[\omega\left(f, \frac{1}{n}\right)+\|f\|_{\infty} \frac{\ln n}{n}\right] .
\end{aligned}
$$

As a result, by the above estimates, we have:

$$
\delta_{n} \leqslant M\left(\omega\left(f, \frac{1}{n}\right)+\|f\|_{\infty} \frac{\ln n}{n}\right) .
$$

The proof is complete.

Theorem 3.2 implies immediately the following corollary.

Corollary 3.1. Let $x_{0} \in \mathbb{R}^{2} \backslash \bar{D}$ and $z_{*}^{n}=\left(z_{1}^{*}, z_{2}^{*}, \ldots, z_{n}^{*}\right)^{\mathrm{T}}$ is a solution to system of algebraic equations 3.1. Then the sequence

$$
\begin{aligned}
u_{n}\left(x_{0}\right)= & \frac{b-a}{n} \sum_{j=1}^{n} \Phi\left(x_{0}, x\left(\tau_{j}\right)\right) \sqrt{\left(x_{1}^{\prime}\left(\tau_{j}\right)\right)^{2}+\left(x_{2}^{\prime}\left(\tau_{j}\right)\right)^{2}} z_{j}^{*}+i \mu\left(\frac{b-a}{n}\right)^{2} \sum_{j=1}^{n} \frac{\partial \Phi\left(x_{0}, x\left(\tau_{j}\right)\right)}{\partial \vec{n}\left(x\left(\tau_{j}\right)\right)} \\
& \cdot\left(\sum_{\substack{m=1 \\
m \neq j}}^{n} \Phi\left(x\left(\tau_{j}\right), x\left(\tau_{m}\right)\right) \sqrt{\left(x_{1}^{\prime}\left(\tau_{m}\right)\right)^{2}+\left(x_{2}^{\prime}\left(\tau_{m}\right)\right)^{2}} z_{m}^{*}\right) \sqrt{\left(x_{1}^{\prime}\left(\tau_{j}\right)\right)^{2}+\left(x_{2}^{\prime}\left(\tau_{j}\right)\right)^{2}}
\end{aligned}
$$


converges to the value $u\left(x_{0}\right)$ at the point $x_{0}$ of the solution $u(x)$ of mixed boundary value problem for the Laplace equation and

$$
\left|u_{n}\left(x_{0}\right)-u\left(x_{0}\right)\right| \leqslant M\left(\omega\left(f, \frac{1}{n}\right)+\|f\|_{\infty} \frac{\ln n}{n}\right) .
$$

\section{BIBLIOGRAPHY}

1. R.J. Heydarov. On solvability of an external problem with impedance boundary condition for Helmholtz equation by integral equations method // Proc. Inst. Math. Mech. NAS Azerbaijan. 42:1, 3-9 (2016).

2. E.H. Khalilov. Constructive method for solving a boundary value problem with impedance boundary condition for the Helmholtz equation // Differ. Uravn. 54:4, 544-555 (2018). [Diff. Equat.

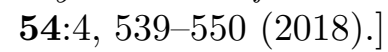

3. E.G. Khalilov. justification of the collocation method for a class of surface integral equations // Matem. Zamet. 107:4, 604-622 (2020). [Math. Notes. 107:4, 663-678 (2020).]

4. E.H. Khalilov. On approximate solution of external Dirichlet boundary value problem for Laplace equation by collocation method // Azerbaijan J. Math. 5:2, 13-20 (2015).

5. R. Kress. Boundary integral equations in time-harmonic acoustic scattering // Math. Comput. Model. 15: 3-5, 229-243 (1991).

6. N.I. Muskhelishvili. Singular integral equations. Fiz.-Mat. Liter., Moscow (1962). [WoltersNoordhoff Publ., Groningen (1967).]

7. V.S. Vladimirov. Equations of mathematical physics. Nauka, Moscow (1976). [Marcel Dekker, New York (1971).]

8. E.H. Khalilov, M.N. Bakhshaliyeva. Quadrature formulas for simple and double layer logarithmic potentials // Proc. Inst. Math. Mech. NAS Azerbaijan. 45:1, 155-162 (2019).

9. Yu.A. Kustov, B.I. Musaev. Cubature formula for two-dimensional singular integral and its applications // Preprint no. 4281-81, VINITI 60 pp. (1981). (in Russian).

10. D. Colton, R. Kress. Integral equation methods in scattering theory. John Wilry \& Sons, New York (1983).

11. G.M. Vainikko. Regular convergence of operators and the approximate solution of equations // Itogi Nauki Tekh. Matem. Anal. 16, 5-53 (1979). [J. Soviet Math. 15:6, 675-705 (1981).]

Khalilov Elnur Hasan ogly,

Azerbaijan State Oil and Industrial University,

Azadliq av. 20,

AZ 1010, Baku, Azerbaijan

E-mail: elnurkhalil@mail.ru

Bakhshaliyeva Mekhpara Nusrat kyzy,

Azerbaijan State Oil and Industrial University,

Azadlıq av. 20,

AZ 1010, Baku, Azerbaijan

E-mail: mehpara.bakhshalieva@mail.ru 